\title{
Invasion patterns in stage I endometrioid and mucinous ovarian carcinomas: a clinicopathologic analysis emphasizing favorable outcomes in carcinomas without destructive stromal invasion and the occasional malignant course of carcinomas with limited destructive stromal invasion
}

\author{
Shirley Chen ${ }^{1}$, Mario M Leitao ${ }^{2}$, Carmen Tornos $^{1}$ and Robert A Soslow ${ }^{1}$ \\ ${ }^{1}$ Department of Pathology, Memorial Sloan-Kettering Cancer Center, New York, NY, USA and ${ }^{2}$ Gynecology \\ Service, Department of Surgery, Memorial Sloan-Kettering Cancer Center, New York, NY, USA
}

\begin{abstract}
Stage I, low-grade endometrioid and mucinous ovarian carcinomas have an excellent prognosis. Published data have suggested that destructive stromal invasion, a relatively uncommon finding in these tumors, is a poor prognostic factor. We investigated this by studying all FIGO stage I, grades 1 and 2 (of 3) endometrioid and mucinous ovarian carcinomas that were surgically staged at the Memorial Sloan-Kettering Cancer Center from 1980 to 2000. We undertook a careful review of all available slides using current diagnostic criteria and correlated histopathologic indices with clinical outcome data. Cases studied included 13 endometrioid ovarian carcinomas (stage IA, eight; stage IC, five) and six intestinal mucinous ovarian carcinomas (stage IA, three; stage IC, three). All of the tumors contained areas of expansile invasion, greater than that acceptable for microinvasion, and were thus diagnosed as carcinomas instead of borderline tumors. Nevertheless, nearly all demonstrated borderline tumor (noninvasive) components. Six tumors contained at least one focus of destructive stromal invasion (two endometrioid and four mucinous ovarian carcinomas). Four additional cases showed a focus suspicious for but not diagnostic of destructive invasion ('indeterminate for destructive invasion') (two endometrioid and two mucinous ovarian carcinomas). Follow-up data were available for 17 patients. The median follow-up was 81 months (range, 9-161 months). In all, 14 patients were alive with no evidence of disease (expansile invasion alone, eight; destructive stromal invasion, four; and indeterminate for destructive invasion, two). Three patients died of their disease (destructive stromal invasion, two; and indeterminate for destructive invasion, one). The size, number, and nuclear grade of destructive stromal invasion foci did not appear to have an impact on survival in this relatively limited number of patients. Outcome data in patients with stage I, low-grade endometrioid and mucinous ovarian carcinomas without destructive stromal invasion indicate that these tumors have a very limited malignant potential. The literature has not documented recurrences in this setting when the staging has been complete, the sampling adequate, and the cytologic features no more than grade 2, and metastasis to the ovary has been excluded. In contrast, carcinomas harboring even limited foci of destructive stromal invasion, although frequently cured surgically, can pursue a malignant clinical course.

Modern Pathology (2005) 18, 903-911. doi:10.1038/modpathol.3800366; Published online 14 January 2005
\end{abstract}

Keywords: endometrioid; mucinous; ovary; ovarian carcinoma; invasion; expansile invasion; destructive invasion

Correspondence: Dr RA Soslow, MD, Department of Pathology, Memorial Sloan-Kettering Cancer Center, 1275 York Avenue, C-524 New York, NY 10021, USA.

E-mail: soslowr@mskcc.org

Received 25 August 2004; revised 17 November 2004; accepted 19

November 2004; published online 14 January 2005
Unlike serous carcinomas, ovarian mucinous and endometrioid adenocarcinomas mostly present as low-stage tumors and tend to have a favorable prognosis. Another possible explanation for these tumors' mostly indolent behavior, particularly at the well-differentiated end of the spectrum, is their 
histologic and biologic overlap with mucinous and endometrioid borderline tumors. In fact, most welldifferentiated mucinous ovarian adenocarcinomas and endometrioid adenocarcinomas show mostly expansile invasion or a confluent glandular growth pattern without destructive stromal invasion. Expansile invasion, first formally described in mucinous adenocarcinomas, ${ }^{1}$ refers to architecturally complex glandular arrangements incompatible with a noninvasive process.

There have been numerous clinicopathologic reports of mucinous ovarian adenocarcinomas and endometrioid adenocarcinomas, ${ }^{1-19}$ but none have focused specifically on the importance of invasion patterns. Three relatively recent publications, two dealing with mucinous ovarian adenocarcinomas ${ }^{1,17}$ and one with endometrioid adenocarcinomas, ${ }^{2}$ have described in detail histologic patterns of invasion, but these papers primarily emphasized criteria distinguishing borderline tumors from carcinomas and primary ovarian carcinomas from metastases.

Our data include a detailed histologic analysis of stromal invasive patterns and their relevance in a group of well-characterized, surgically and pathologically staged tumors with clinical follow-up information. Mucinous ovarian adenocarcinomas and endometrioid adenocarcinomas were grouped together in this study because of their generally similar architectural features, invasion patterns, and clinical outcomes. The purpose of this study was to determine the impact of destructive stromal invasion on outcome in patients with stage I, low-grade (grades 1 and 2 (of 3)) mucinous ovarian adenocarcinomas and endometrioid adenocarcinomas.

\section{Methods}

We reviewed all FIGO stage I, grades 1 and 2 (of 3) mucinous ovarian adenocarcinomas and endometrioid adenocarcinomas that were surgically staged at the Memorial Sloan-Kettering Cancer Center (MSKCC) from 1980 to 2000. Among mucinous ovarian adenocarcinomas, only intestinal mucinous ovarian carcinomas were studied. Mullerian mucinous carcinomas and mucinous tumors associated with pseudomyxoma peritonei syndrome were excluded. The criteria for adequate staging included omentectomy, peritoneal biopsies, and evaluation of lymph nodes by inspection and palpation. Comprehensively staged patients underwent lymph node sampling or dissection with pathologic evaluation. We retrieved a total of 19 cases (13 endometrioid adenocarcinomas and six mucinous ovarian adenocarcinomas) that were suitable for the study. Patients diagnosed after $1990(n=14)$ underwent comprehensive staging; the remainder $(n=5)$ underwent adequate staging. This material constituted a subgroup of cases that were the subject of a previously published study that presented the clinicopathologic features of low-stage ovarian carcinomas of all histologic types. ${ }^{20}$ The significance of invasion patterns in mucinous ovarian adenocarcinoma and endometrioid adenocarcinoma was not detailed in the previous publication. The number of slides per case examined ranged from 7 to 27 (median, 16 slides). Histologic features examined included invasion type (destructive stromal invasion vs expansile invasion), number of destructive stromal invasion foci, grade of destructive stromal invasion foci, and size of destructive stromal invasion foci.

All of the tumors contained areas of expansile invasion greater than that acceptable for microinvasion and were thus diagnosed as carcinomas instead of borderline tumors. We did not use the term 'microinvasion' to describe destructive stromal invasion foci because all of the tumors studied were diagnostic of carcinoma by virtue of extensive expansile invasion; none were borderline tumors, the context in which the term 'microinvasion' has been used. We adapted the endometrial carcinoma criteria of Longacre et $a l^{21}$ to recognize expansile invasion. Briefly, expansile invasion areas were characterized by one or more of the following criteria: (1) glands with extensive branching and budding, with concomitant exclusion of intervening stroma; (2) large glands (macroglands) with substantial internal complexity (true cribriform architecture); (3) complicated folia or secondary papillary structures superimposed on folia; or (4) abundant, exophytic coarse and fine papillae (Figure 1). Destructive stromal invasion of any size was diagnosed when there was at least one of the following: (1) unequivocal disruption of a basement membrane by jagged, irregularly spaced, and unevenly shaped tumor cell nests in stroma, with an edematous and/or inflamed stromal response; or (2) groups of single neoplastic cells in stroma, usually with an accompanying stromal response (Figure 2). If there were any questions about whether destructive stromal invasion had occurred, either because of one observer's doubts or a lack of consensus between two observers, the opinion of another pathologist was sought. All such cases were ultimately scored as indeterminate for destructive invasion (Figure 3).

We adhered to the FIGO grading scheme used for endometrial carcinoma for assigning overall tumor grade, which was determined using an architectural (original) grade and a nuclear grade. Just as in endometrial cancer, the presence of grade 3 nuclei, whether in expansile invasion or destructive stromal invasion foci, changed an original grade 1 tumor to one that was overall grade 2 , or from a grade 2 tumor to one that was overall grade 3 . Nuclear grade 1 tumor cell nuclei resembled those of a borderline tumor or nuclear grade 1 endometrioid adenocarcinoma of endometrium. Grade 2 nuclei showed moderate variation in nuclear size (up to three times variation), somewhat irregular nuclear outlines, and 

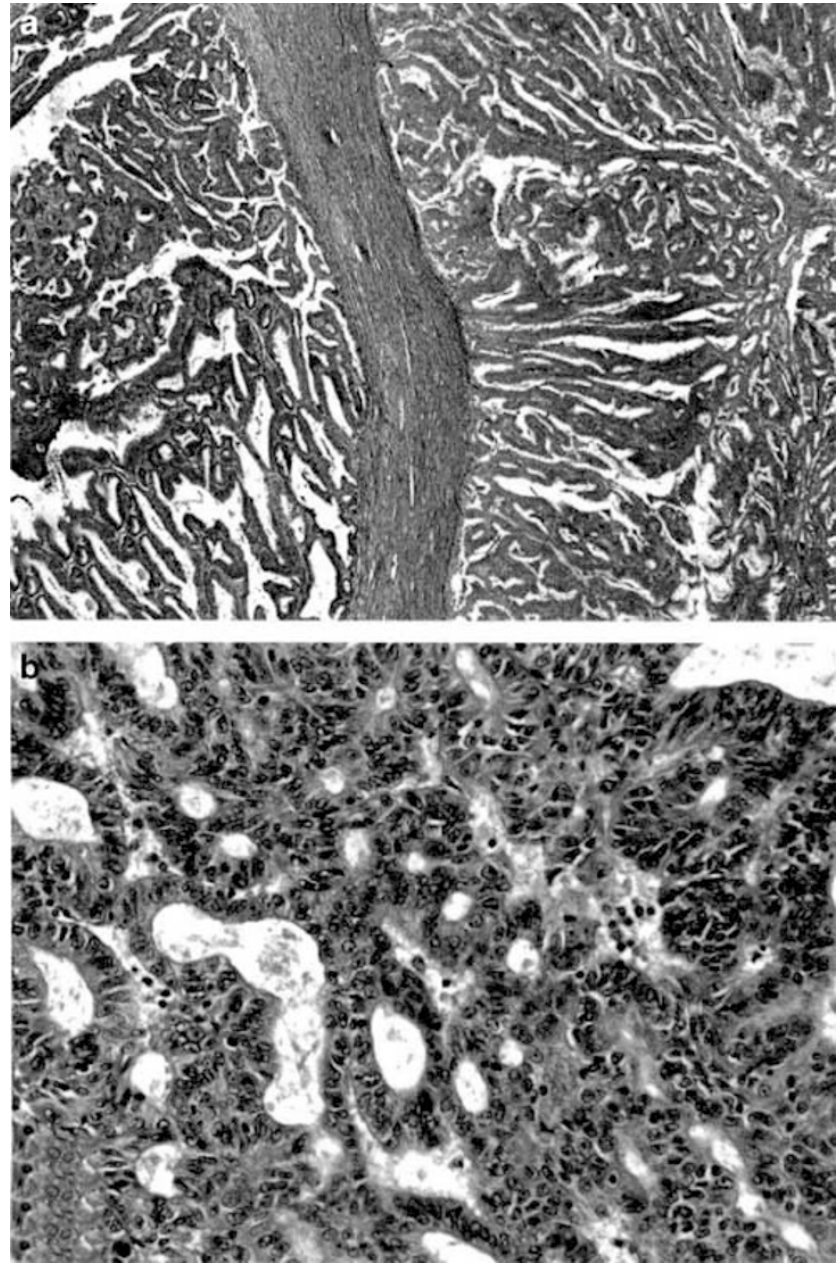

Figure 1 Expansile invasion in endometrioid adenocarcinoma: most tumors had at least a focal borderline component and all cases showed expansile invasion greater than microinvasion, illustrated in (a and b). Borderline components differed from expansile invasive carcinomas by the absence of substantial architectural complexity. Examples of architecturally complex proliferations (carcinomas) are shown in panels (a) (extensively branched and budded glands without intervening stroma) and (b) (true cribriform structures).

nucleoli that were only prominent on high power ( $>\times 200$ magnification). Grade 3 nuclei showed variation in nuclear size greater than three times, markedly irregular nuclear contours, and prominent nucleoli, all obvious at intermediate power magnification $(\times 100-200)$.

Clinical data were abstracted from clinical charts. Charts from patients with mucinous ovarian adenocarcinoma were specifically searched for data indicating or suggesting whether the patient's ovarian tumor represented a metastasis. Cases for which doubt existed about an ovarian primary were excluded. All cases with features of a metastasis to the ovary were excluded. ${ }^{22,23}$ Furthermore, a representative section of each mucinous ovarian adenocarcinoma was analyzed with CK7 and CK20 antibodies, as described previously. ${ }^{20}$ Any tumor showing CK20 expression without any CK7 expression was also excluded.

\section{Results}

\section{Gross Pathology}

Nearly all tumors were multicystic, with varying degrees of solid components, either interposed between cysts (seen in many mucinous tumors) or projecting inside cysts (seen in many endometrioid tumors). The ovarian masses ranged in size from 4 to $30 \mathrm{~cm}$ in greatest dimension (mean, $14 \mathrm{~cm}$ ). Four endometrioid adenocarcinomas contained cysts with coarse papillary projections. Necrosis and hemorrhage were seen in less than half of the cases (7/19). Only one case was bilateral-an endometrioid adenocarcinoma, stage IC. Ovarian surface involvement was also described in one case.

\section{Microscopic Pathology}

The data are summarized in Table 1 . There were eight stage IA endometrioid adenocarcinomas and five stage IC endometrioid adenocarcinomas. Three stage IC endometrioid adenocarcinomas had ovarian surface involvement; the remainder were ruptured intraoperatively. There were 10 histologic grade 1 endometrioid adenocarcinomas (eight showing grade 1 nuclei and two showing grade 2 nuclei) and three histologic grade 2 endometrioid adenocarcinomas (two with grade 1 nuclei and one with grade 2 nuclei).

There were three stage IA mucinous ovarian adenocarcinomas and three stage IC mucinous ovarian adenocarcinomas. Two of the stage IC mucinous ovarian adenocarcinomas were ruptured preoperatively and one was ruptured intraoperatively. None of the mucinous ovarian adenocarcinomas showed ovarian surface involvement. There were three histologic grade 1 mucinous ovarian adenocarcinomas (two showing grade 1 nuclei and one showing grade 2 nuclei) and three histologic grade 2 mucinous ovarian adenocarcinomas (one with grade 1 nuclei and two with grade 3 nuclei limited to the destructive stromal invasion component). All mucinous carcinomas expressed CK7 diffusely. CK20 immunoreactivity was generally patchy and weak.

Five patients had associated endometriosis (four endometrioid adenocarcinomas and one mucinous ovarian adenocarcinoma). One endometrioid adenocarcinoma was associated with endometrial hyperplasia and five were associated with FIGO grade 1 endometrioid adenocarcinomas of the endometrium, three of which were noninvasive and two of which showed minimal myometrial invasion (less than $1.5 \mathrm{~mm}$ ). None showed lymphovascular invasion. There were no mucinous ovarian adenocarcinomas associated with endometrial lesions. Six 
906
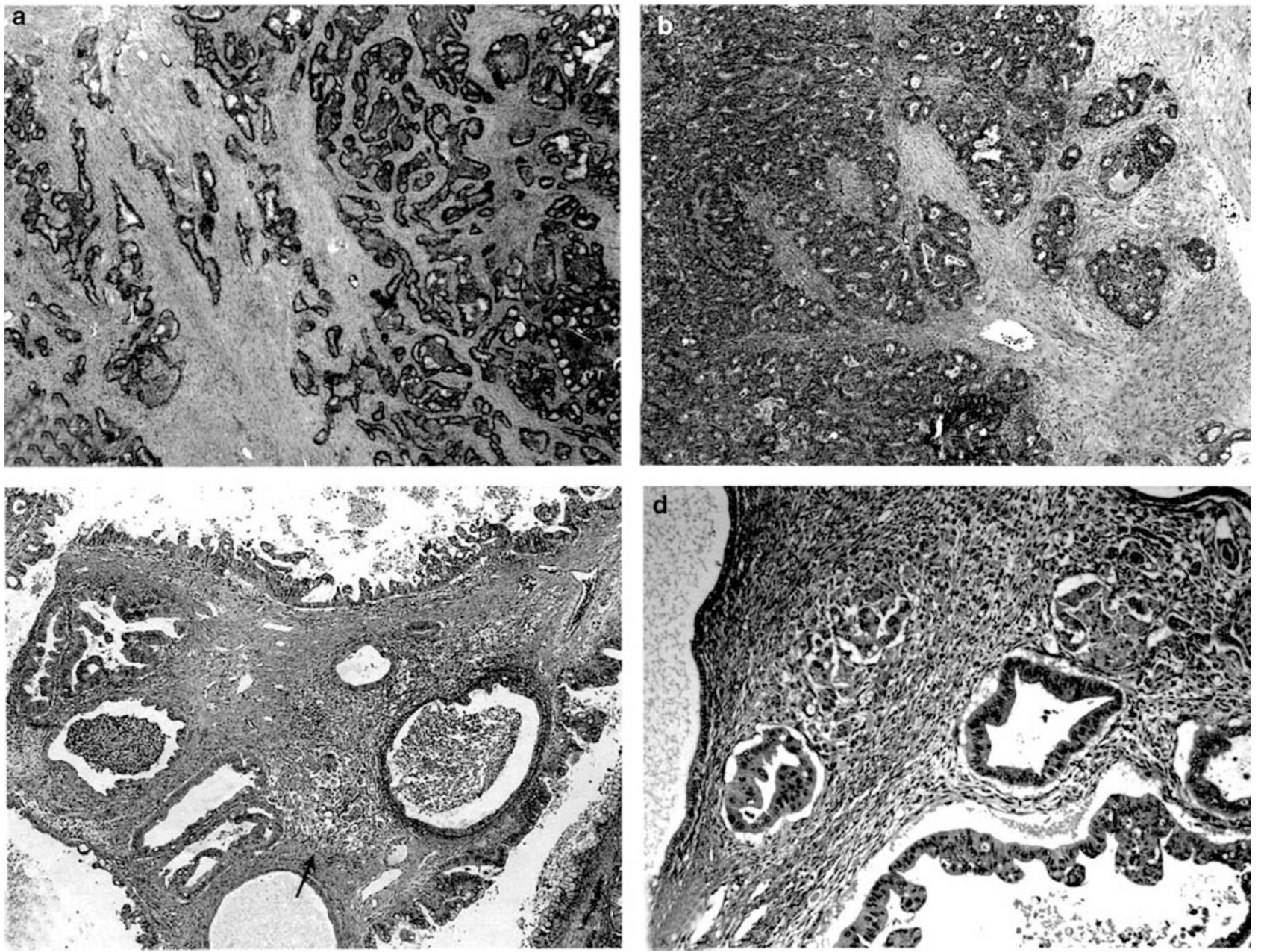

Figure 2 Destructive invasion in endometrioid adenocarcinoma (a and $\mathbf{b}$ ) and mucinous adenocarcinoma (c and $\mathbf{d}$ ): examples of pointed glands infiltrating altered stroma (a), rounded glands infiltrating altered stroma (b), and groups of eosinophilic cells with a surrounding retraction space at low power (see arrow) (c) and high power (d) are shown.

endometrioid adenocarcinomas had associated benign ovarian adenofibromatous components, six showed more than focal squamous differentiation, and two endometrioid adenocarcinomas showed more than focal mucinous differentiation. One endometrioid adenocarcinoma contained extensive sex-cord-like areas.

Each tumor contained areas of expansile invasion (Figure 1), and nearly all demonstrated borderline tumor (noninvasive) components. In seven tumors, a borderline component represented only a small fraction $(<25 \%)$ of the neoplasm. In three tumors, the noninvasive component area approximated that of expansile invasion, and in eight tumors, noninvasive components predominated; in three of the latter cases, expansile invasion areas were focal or multifocal and measured between 5 and $10 \mathrm{~mm}$. All tumors but two mucinous ovarian adenocarcinomas studied showed glands with extensive branching and budding, with concomitant exclusion of intervening stroma and glands with substantial internal complexity (true cribriforming). In these two muci- nous ovarian adenocarcinomas, true cribriform structures were not identified; they exhibited complicated folia instead. One case, another mucinous ovarian adenocarcinoma, showed glands with extensive budding, large glands with notable internal complexity, and complicated folia.

Six tumors contained at least one destructive stromal invasion focus (two endometrioid adenocarcinomas and four mucinous ovarian adenocarcinomas) and four showed an indeterminate for destructive invasion focus (two endometrioid adenocarcinomas and two mucinous ovarian adenocarcinomas) (Figures 2 and 3). The destructive stromal invasion foci in the endometrioid adenocarcinomas were composed mostly of pointed single glands, irregularly dispersed in edematous, desmoplastic stroma containing lymphocytes. The constituent cells frequently showed increased cytoplasmic eosinophilia relative to the expansile invasion foci. The destructive stromal invasion foci in the mucinous ovarian adenocarcinomas showed rounded glands and single cells set in an inflamed and 

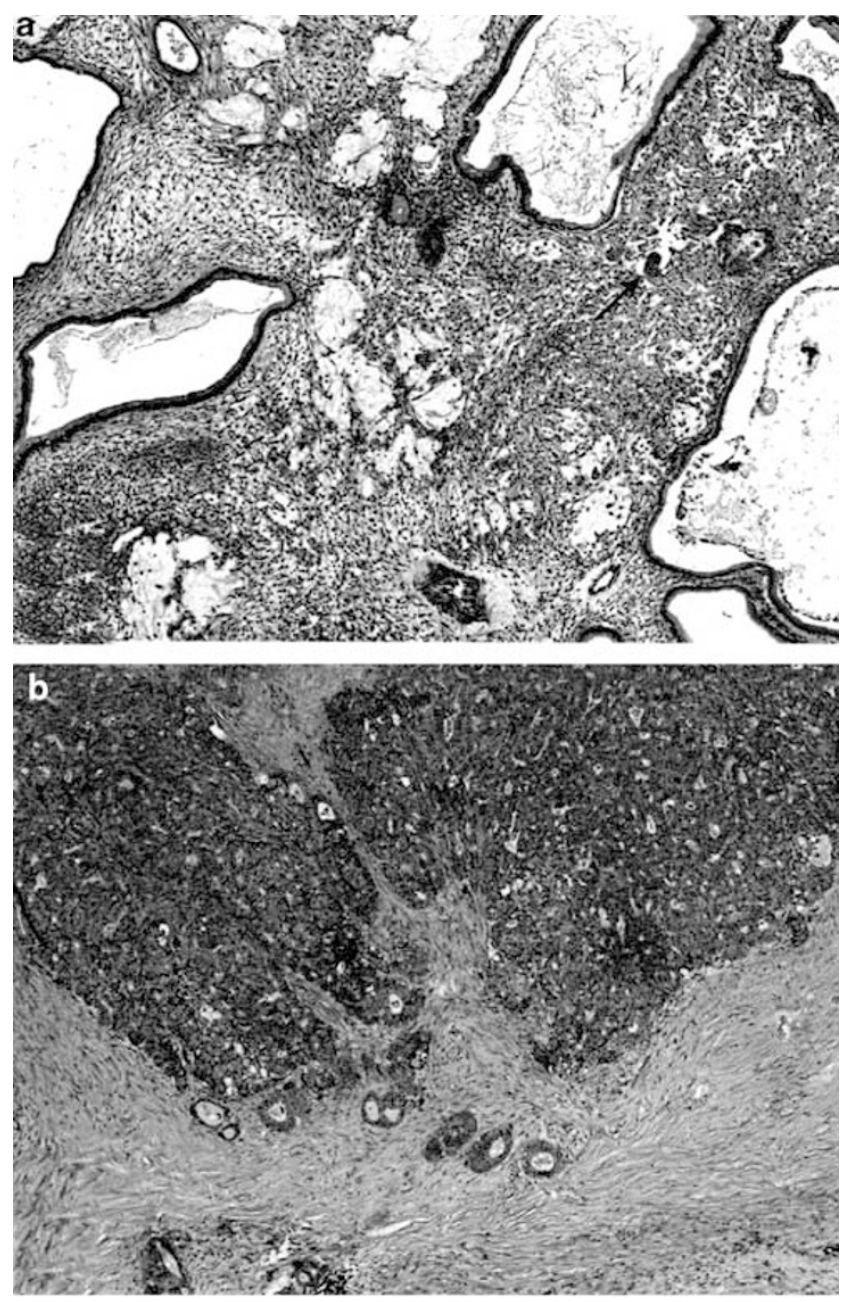

Figure 3 Indeterminate for invasion: One mucinous adenocarcinoma indeterminate for destructive invasion showed patchy stromal mucin deposition in which rare, bland epithelial cells (arrow) were found (a). Another case indeterminate for invasion, an endometrioid adenocarcinoma, showed contour irregularities at the periphery of an otherwise rounded lobule of expansile invasion (b).

desmoplastic stroma containing neutrophils. Here too, the destructive stromal invasion foci contained cells with increased cytoplasmic eosinophilia and less prominent intracytoplasmic mucin relative to expansile invasion foci.

The appearances of the indeterminate for destructive invasion foci took one of three forms (Figure 3): rare, irregularly shaped mucin pools in which scant dissociated bland epithelial cells floated, suggesting gland rupture; contour irregularities at the periphery of large expansile invasion foci that were otherwise rounded; or adenofibromatous tumors (particularly endometrioid adenocarcinomas) in which single glands without pointed profiles were set in noninflamed fibrous stroma between rounded lobules of expansile invasion. The number, size, and nuclear grade of the destructive stromal invasion and indeterminate for destructive invasion foci are detailed in Table 1. The nuclear grade of each destructive stromal invasion focus was the same as in the expansile invasion areas in two cases, and exceeded that in expansile invasion areas in four cases.

\section{Clinical Correlation}

Follow-up was available for 17 patients. The median follow-up time was 81 months (range, 9-161 months). In all, 11 patients were treated with systemic chemotherapy and one was treated with intraperitoneal platinum and intravenous paclitaxel. Nine patients, all treated systemically, received a platinum-based regimen in addition to a taxane. Six patients, all with stage IA tumors, did not receive adjuvant therapy for ovarian carcinoma.

A total of 14 patients were alive with no evidence of disease (NED)—eight patients with expansile invasion alone, four with destructive stromal invasion, and two indeterminate for destructive invasion. Three patients died of disease (DOD) - two with destructive stromal invasion (both mucinous ovarian adenocarcinomas) and one indeterminate for destructive invasion (an endometrioid adenocarcinoma). One mucinous ovarian adenocarcinoma was stage IA and the other was stage IC. Both tumors were architectural grade 1 and nuclear grade 1 in expansile invasion areas. We found two destructive stromal invasion foci in each tumor; both measured only $1 \mathrm{~mm}$. In one case, the destructive stromal invasion foci showed grade 3 nuclear atypia, and in the other, the destructive stromal invasion foci showed grade 2 nuclear atypia. The indeterminate for destructive invasion case, a stage IC, histologic grade 1 and nuclear grade 1 endometrioid adenocarcinoma, exhibited adenofibromatous architecture in which neoplastic-appearing glands were found in fibromatous stroma between rounded lobules of expansile invasion.

Table 2 shows a summary of findings comparing tumors from DOD patients with tumors from NED patients. Two of three DOD cases were stage IC, showed destructive stromal invasion, and exhibited mucinous histology. Table 3 displays data comparing destructive stromal invasion cases in DOD vs NED patients. The one patient's tumor that showed only one focus of destructive stromal invasion was NED. The destructive stromal invasion foci in DOD patients' tumors each measured only $1 \mathrm{~mm}$, whereas such foci in NED tumors were paradoxically more extensive. None of the DOD patients with destructive stromal invasion had endometrioid pathology.

The DOD patient with stage IA mucinous ovarian adenocarcinoma recurred with poorly differentiated adenocarcinoma in the liver and pleura 21 months after diagnosis and succumbed to disease 23 months after diagnosis. The DOD patient with stage IC mucinous ovarian adenocarcinoma developed biopsy-proven metastatic well-differentiated adenocarcinoma in the lung 34 months after diagnosis and 
Table 1 Data summary

\begin{tabular}{|c|c|c|c|c|c|c|c|c|}
\hline $\begin{array}{l}\text { Histo } \\
\text { subtype }\end{array}$ & Stage & $\begin{array}{l}\text { Invasion } \\
\text { type }\end{array}$ & Number of DI foci & $\begin{array}{l}\text { Size } \\
\text { of DI }\end{array}$ & $\begin{array}{l}\text { Overall grade/ } \\
\text { nuclear grade }\end{array}$ & $\begin{array}{l}\text { DI nuclear } \\
\text { grade }\end{array}$ & $\begin{array}{l}\text { Outcome/PFI } \\
\text { (months) }\end{array}$ & $\begin{array}{c}\text { PFI } \\
\text { (months) }\end{array}$ \\
\hline EC & IA & EI & None & N/A & $1 / 1$ & N/A & NED & 61 \\
\hline EC & IA & EI & None & N/A & $1 / 1$ & N/A & NED & 38 \\
\hline EC & IA & EI & None & N/A & $2 / 1$ & N/A & NED & 6 \\
\hline EC & IA & EI & None & N/A & $1 / 1$ & N/A & N/A & N/A \\
\hline EC & IA & EI & None & N/A & $2 / 1$ & N/A & NED & 65 \\
\hline EC & IA & EI & None & N/A & $1 / 1$ & N/A & NED & 69 \\
\hline EC & IA & DI & 3 & $2,4,6 \mathrm{~mm}$ & $1 / 2$ & 2 & NED & 39 \\
\hline EC & IA & DI & $>5$ & $1-7 \mathrm{~mm}$ & $1 / 2$ & 2 & NED & 22 \\
\hline EC & IC & EI & None & N/A & $1 / 1$ & N/A & NED & 41 \\
\hline EC & IC & EI & None & N/A & $1 / 1$ & N/A & NED & 37 \\
\hline EC & IC & EI & None & N/A & $1 / 1$ & N/A & NED & 47 \\
\hline EC & IC & IDI & None & N/A & $1 / 1$ & N/A & DOD & 12 \\
\hline EC & IC & IDI & None & $\mathrm{N} / \mathrm{A}$ & $1 / 2$ & N/A & N/A & N/A \\
\hline MC & IA & DI & 2 & $1 \mathrm{~mm}$ & $2 / 1$ & 2 & NED & 41 \\
\hline MC & IA & DI & 1 & $1 \mathrm{~mm}$ & $2 / 2$ & 3 & NED & 67 \\
\hline MC & IA & DI & 3 & $1 \mathrm{~mm}$ & $2 / 1$ & 3 & DOD & 9 \\
\hline MC & IC & DI & 2 & $1 \mathrm{~mm}$ & $1 / 1$ & 2 & DOD & 34 \\
\hline MC & IC & IDI & None & N/A & $1 / 1$ & N/A & NED & 33 \\
\hline MC & IC & IDI & None & N/A & $1 / 2$ & N/A & NED & 135 \\
\hline
\end{tabular}

EC, endometrioid carcinoma; MC, mucinous carcinoma; Histo, histologic; EI, expansile invasion alone; DI, destructive invasion; IDI, indeterminate invasion; N/A, not applicable; PFI, progression-free interval; NED, no evidence of disease; DOD, dead of disease.

${ }^{\mathrm{a}}$ Nuclear grade of EI foci. DI nuclear grade is tabulated separately.

Table 2 Tumor characteristics versus clinical outcome

\begin{tabular}{lcc}
\hline & $N E D(\mathrm{n}=14)$ & $D O D(\mathrm{n}=3)$ \\
\hline MC & $4 / 14$ & $2 / 3$ \\
EC & $10 / 14$ & $1 / 3$ \\
DI & $4 / 14$ & $2 / 3$ \\
IDI & $2 / 14$ & $1 / 3$ \\
EI only & $8 / 14$ & $0 / 3$ \\
Stage IA & $9 / 14$ & $1 / 3$ \\
Stage IC & $5 / 14$ & $2 / 3$ \\
Grade 1 & $10 / 14$ & $2 / 3$ \\
Grade 2 & $4 / 14$ & $1 / 3$ \\
\end{tabular}

MC, mucinous carcinoma; EC, endometrioid carcinoma; DI, destructive invasion; IDI, indeterminate invasion; EI, expansile invasion alone; NED, no evidence of disease; DOD, dead of disease.

Table 3 DI tumor characteristics versus clinical outcome

\begin{tabular}{lcc}
\hline & $N E D(\mathrm{n}=4)$ & $D O D(\mathrm{n}=2)$ \\
\hline MC & $2 / 4$ & $2 / 2$ \\
EC & $2 / 4$ & $0 / 2$ \\
Stage IA & $4 / 4$ & $1 / 2$ \\
Stage IC & $0 / 4$ & $1 / 2$ \\
Grade 1 & $2 / 4$ & $1 / 2$ \\
Grade 2 & $2 / 4$ & $1 / 2$ \\
DI grade 2 & $3 / 4$ & $1 / 2$ \\
DI grade 3 & $1 / 4$ & $1 / 2$ \\
DI focus =1 & $1 / 4$ & $0 / 2$ \\
DI foci $>1$ & $3 / 4$ & $2 / 2$ \\
\hline
\end{tabular}

MC, mucinous carcinoma; EC, endometrioid carcinoma; DI, destructive invasion; EI, expansile invasion alone; NED, no evidence of disease; DOD, dead of disease. died 48 months after diagnosis. The DOD patient with stage IC endometrioid adenocarcinoma developed biopsy-proven peritoneal carcinomatosis 22 months after diagnosis and then pleural disease, diagnosed in a thoracentesis specimen the month after that; she died 24 months after diagnosis.

\section{Discussion}

Low-stage ovarian cancers are a heterogeneous group composed of various tumors, each of which possesses distinctive biologic and morphologic attributes. The literature reports several indices of possible prognostic importance, including TP53 mutation, clear cell histology, and high grade and high substage, although recently only TP53 mutation was found to correlate with outcome. ${ }^{20,24}$ Stage I endometrioid adenocarcinomas and mucinous ovarian adenocarcinomas only infrequently demonstrate these poor prognostic features and, on the whole, are prognostically favorable tumors. One of our goals was to discern whether destructive invasion constituted a poor prognostic indicator.

Numerous factors have contributed to our imperfect understanding of these tumors and called into question the results of some previous clinical outcome studies. Patients with low-stage disease have, until recently, only uncommonly undergone a comprehensive staging procedure. The criteria separating endometrioid and mucinous borderline tumors from carcinomas have undergone significant changes, ${ }^{1,2,17,20}$ which might have affected outcome 
data in the older literature; $;^{3-16,18,19}$ furthermore, the more recently proposed criteria, while significantly better than those previously used, can be difficult to apply. Also, as is now widely appreciated, many mucinous tumors, particularly with mostly borderline or only focally malignant features, represent metastases to ovaries, not primary ovarian mucinous neoplasms. ${ }^{25-27}$ Finally, the impact of destructive stromal invasion, as opposed to expansile invasion, has only recently been noted as a possible prognostic factor. ${ }^{1,2,17}$

We report here the clinicopathologic features of all stage I, grades 1 and 2 endometrioid adenocarcinomas and mucinous ovarian adenocarcinomas well staged at MSKCC during a 20-year period. We used the most recently proposed criteria, ${ }^{1,2,17}$ supplemented with those of Longacre et $a l^{21}$ for distinguishing these from borderline tumors and have designed the study to elucidate the significance of destructive stromal invasion with respect to outcome. In the context of invasion patterns, we confirm that all stage I endometrioid adenocarcinomas and mucinous ovarian adenocarcinomas show mostly expansile invasion and that only a subpopulation contain expansile invasion in addition to destructive stromal invasion, the latter of which was almost always microscopic in extent. We did not consider microscopic destructive stromal invasion foci to represent microinvasion since every case contained sufficient expansile invasion for a carcinoma diagnosis.

We did not encounter a single case showing expansile invasion alone that metastasized. Destructive stromal invasion cases were found in both the NED and DOD groups and, based only on our small study numbers, nuclear grade, number of destructive stromal invasion foci, and size of destructive stromal invasion foci, did not correlate with the outcome. Three patients experienced metastases (two with destructive stromal invasion tumors and one tumor indeterminate for destructive invasion). Reviewing the latter case retrospectively suggested to us that the simple glands observed in fibromatous stroma between rounded lobules of expansile invasion probably represented true destructive stromal invasion, although suboptimal sampling that might have obscured a more convincingly invasive focus cannot be entirely excluded.

One could surmise that the two other indeterminates for destructive invasion patterns observed, small patches of mucin dissection adjacent to expansile invasion foci and contour irregularities at the periphery of an expansile invasion focus, might not signify true destructive stromal invasion, but our data on this point are clearly scant and thus preliminary. The problem posed by mucin dissection has been described previously. ${ }^{1,10,12,17}$ There are several correlates in other organs: benign mucocelelike lesions in breast and mucin granulomas in inflammatory bowel disease, among others. We think that invasive carcinoma should not be diagnosed when the gland from which the mucin has spilled is lined by benign-appearing epithelium and when the epithelium sloughed into the extravasated mucin is similarly morphologically bland. This situation should be distinguished from pseudomyxoma ovarii, in which there are extensive and large pools of mucin, and from invasive mucinous carcinomas that almost always contain cytologically malignant epithelium and unequivocal jagged stromal invasion in areas away from mucin dissection.

The destructive stromal invasion patterns associated with carcinomas described herein resemble the occasional destructive stromal invasion foci seen in endometrioid and intestinal mucinous borderline tumors, also known as microinvasion. $^{1,2,12,17}$ Despite the histologic similarities between destructive stromal invasion in borderline tumors and destructive stromal invasion in carcinomas, the clinical significance probably differs. Kurman's group, in particular, has reported that borderline tumors with less than $5 \mathrm{~mm}$ of destructive stromal invasion pursue a benign course. ${ }^{17}$ In contrast, our data, which center on destructive stromal invasion in carcinomas specifically, demonstrate that endometrioid adenocarcinomas and mucinous ovarian adenocarcinomas with even microscopic destructive stromal invasion can pursue a malignant clinical course.

Although there are numerous articles in the literature that discuss the clinicopathologic features of endometrioid and mucinous carcinomas, ${ }^{3-16,18,19}$ most do not specify the type of invasion required to diagnose carcinoma. The study by de Nictolis et $a{ }^{7}{ }^{7}$ however, reports that destructive stromal invasion was not seen in any stage IA mucinous ovarian adenocarcinoma. Survival was $100 \%$ in this study. Three relatively recent publications concerning mucinous ovarian adenocarcinomas have provided an in-depth analysis of criteria differentiating borderline tumors from carcinomas and primary ovarian carcinomas from metastases. ${ }^{1,12,17}$ Included in two of these publications are references to invasion patterns. ${ }^{1,17}$ All carcinomas contained areas with a 'confluent glandular pattern'17 or 'expansile invasion', ${ }^{1}$ presumably synonymous terms. Other invasion patterns described were destructive invasion, seen rarely in primary ovarian mucinous adenocarcinoma and more commonly in metastatic carcinoma, and a nodular invasion pattern, essentially restricted to metastases. Lee and Scully's paper ${ }^{1}$ included 15 stage I, grade 1-3 mucinous ovarian adenocarcinoma patients with follow-up data, representing a mixture of consultation and 'hospital cases.' The mucinous ovarian adenocarcinomas studied, 25 cases in all, were approximately evenly divided between tumors with expansile invasion only and those with expansile invasion and destructive stromal invasion. Only one patient with a clinical stage I mucinous ovarian adenocarcinoma experienced recurrence and died. This patient was suboptimally staged, and her tumor showed intraepithelial carcinoma, expansile 
invasion, and destructive stromal invasion. No stage I mucinous ovarian adenocarcinoma with expansile invasion alone recurred. The paper of Riopel et $a l^{17}$ reports 11 stage I mucinous ovarian adenocarcinomas with follow-up data, representing a mixture of consultation and 'hospital cases' and of low- and high-grade tumors. Only one patient died of disease, but the histologic features of this particular patient's tumor and the extent of staging were not presented. The paper of Hoerl and Hart ${ }^{12}$ presents data on 14 stage I mucinous ovarian adenocarcinomas. These authors report two recurrences. However, only patients whose tumors showed 'extensive stromal invasion,' without distinction of expansile invasion from destructive stromal invasion, were included in the study.

The recent work of Bell and Kurman ${ }^{2}$ on endometrioid adenocarcinomas provides a clinicopathologic description of 15 stage I, grade 1-3 endometrioid adenocarcinoma patients; follow-up information was available for seven patients. Only a portion of patients in this series were formally staged. All 15 endometrioid adenocarcinomas showed expansile invasion, while two also showed destructive stromal invasion. One of the seven patients with follow-up succumbed to disease. This patient's tumor showed expansile invasion exclusively, but it was a grade 3 tumor.

Our results suggest that surgically staged, stage I, low-grade endometrioid adenocarcinomas and mucinous ovarian adenocarcinomas without destructive stromal invasion likely have very limited malignant potential; a larger study of similar cases would certainly be informative. We are not aware of reported recurrences in this setting when the staging has been complete, the sampling adequate, and the overall cytologic grade no more than grade 2 , and metastasis to the ovary has been excluded. In contrast, tumors harboring even focal destructive stromal invasion, can be fatal in an appreciable proportion of cases-one-third in this series.

\section{References}

1 Lee KR, Scully RE. Mucinous tumors of the ovary: a clinicopathologic study of 196 borderline tumors (of intestinal type) and carcinomas, including an evaluation of 11 cases with 'pseudomyxoma peritonei'. Am J Surg Pathol 2000;24:1447-1464.

2 Bell KA, Kurman RJ. A clinicopathologic analysis of atypical proliferative (borderline) tumors and welldifferentiated endometrioid adenocarcinomas of the ovary. Am J Surg Pathol 2000;24:1341-1342.

3 Brescia RJ, Dubin N, Demopoulos RI. Endometrioid and clear cell carcinoma of the ovary-factor affecting survival. Int J Gynecol Pathol 1989;8:132-138.

4 Cariker M, Dockerty M. Mucinous cystadenomas and mucinous cystadenocarcinomas of the ovary: a clinical and pathological study of 355 cases. Cancer 1954;7: 302-310.
5 Curling OM, Hudson CN. Endometrioid tumours of the ovary. Br J Obstet Gynaecol 1975;82:405-411.

6 Czernobilsky B, Silverman BB, Mikuta JJ. Endometrioid carcinoma of the ovary: a clinicopathologic study of 75 cases. Cancer 1970;26:1141-1152.

7 de Nictolis M, Montironi R, Tommasoni S, et al. Benign, borderline, and well-differentiated malignant intestinal mucinous tumors of the ovary: a clinicopathologic, histochemical, immunohistochemical, and nuclear quantitative study of 57 cases. Int J Gynecol Pathol 1994;13:10-21.

8 Gray LA, Barnes ML. Endometrioid carcinoma of the ovary. Obstet Gynecol 1967;29:694-701.

9 Grosso G, Raspagliesi F, Baiocchi G, et al. Endometrioid carcinoma of the ovary: a retrospective analysis of 106 cases. Tumori 1998;84:552-557.

10 Guerrieri C, Hogberg T, Wingren S, et al. Mucinous borderline and malignant tumors of the ovary. A clinicopathologic and DNA ploidy study of 92 cases. Cancer 1994;74:2329-2340.

11 Hart WR, Norris HJ. Borderline and malignant mucinous tumors of the ovary. Histologic criteria and clinical behavior. Cancer 1973;31:1031-1045.

12 Hoerl HD, Hart WR. Primary ovarian mucinous cystadenocarcinomas: a clinicopathologic study of 49 cases with long-term follow-up. Am J Surg Pathol 1998;22:1449-1462.

13 Jimenez AM. Endometrioid carcinoma of the ovary. A clinicopathological study of 17 cases. Eur J Gynaec Oncol 1994;15:96-100.

14 Klemi PJ, Gronroos M. Endometrioid carcinoma of the ovary. A clinicopathologic, histochemical, and electron microscope study. Obstet Gynecol 1979;53:572-579.

15 Kline RC, Wharton JT, Atkinson EN, et al. Endometrioid carcinoma of the ovary: retrospective review of 145 cases. Gynecol Oncol 1990;39:337-346.

16 Kurman RJ, Craig JM. Endometrioid and clear cell carcinoma of the ovary. Cancer 1972;29:1653-1664.

17 Riopel MA, Ronnett BM, Kurman RJ. Evaluation of diagnostic criteria and behavior of ovarian intestinaltype mucinous tumors: atypical proliferative (borderline) tumors and intraepithelial, microinvasive, invasive, and metastatic carcinomas. Am J Surg Pathol 1999;23:617-635.

18 Roth LM, Czernobilsky B, Langley FA. Ovarian endometrioid adenofibromatous and cystadenofibromatous tumors: benign, proliferating, and malignant. Cancer 1981;48:1838-1845.

19 Watkin W, Silva EG, Gershenson DM. Mucinous carcinoma of the ovary. Pathologic prognostic factors. Cancer 1992;69:208-212.

20 Leitao Jr MM, Boyd J, Hummer A, et al. Clinicopathologic analysis of early-stage sporadic ovarian carcinoma. Am J Surg Pathol 2004;28:147-159.

21 Longacre TA, Chung MH, Jensen DN, et al. Proposed criteria for the diagnosis of well-differentiated endometrial carcinoma. A diagnostic test for myoinvasion. Am J Surg Pathol 1995;19:371-406.

22 Lee KR, Young RH. The distinction between primary and metastatic mucinous carcinomas of the ovary: gross and histologic findings in 50 cases. Am J Surg Pathol 2003;27:281-292.

23 Seidman JD, Kurman RJ, Ronnet BM. Primary and metastatic mucinous adenocarcinomas in the ovaries: incidence in routine practice with a new approach to improve intraoperative diagnosis. Am J Surg Pathol 2003;27:985-993. 
24 Leitao MM, Soslow RA, Baergen RN, et al. Mutation and expression of the TP53 gene in early stage epithelial ovarian carcinoma. Gynecol Oncol 2004;93: 301-306.

25 Ronnett BM, Kurman RJ, Shmookler BM, et al. The morphologic spectrum of ovarian metastases of appendiceal adenocarcinomas: a clinicopathologic and immunohistochemical analysis of tumors often misinterpreted as primary ovarian tumors or metastatic tumors from other gastrointestinal sites. Am J Surg Pathol 1997;21:1144-1155.
26 Ronnett BM, Kurman RJ, Zahn CM, et al. Pseudomyxoma peritonei in women: a clinicopathologic analysis of 30 cases with emphasis on site of origin, prognosis, and relationship to ovarian mucinous tumors of low malignant potential. Hum Pathol 1995;26:509-524.

27 Ronnett BM, Zahn CM, Kurman RJ, et al. Disseminated peritoneal adenomucinosis and peritoneal mucinous carcinomatosis. A clinicopathologic analysis of 109 cases with emphasis on distinguishing pathologic features, site of origin, prognosis, and relationship to 'pseudomyxoma peritonei'. Am J Surg Pathol 1995;19:1390-1408. 